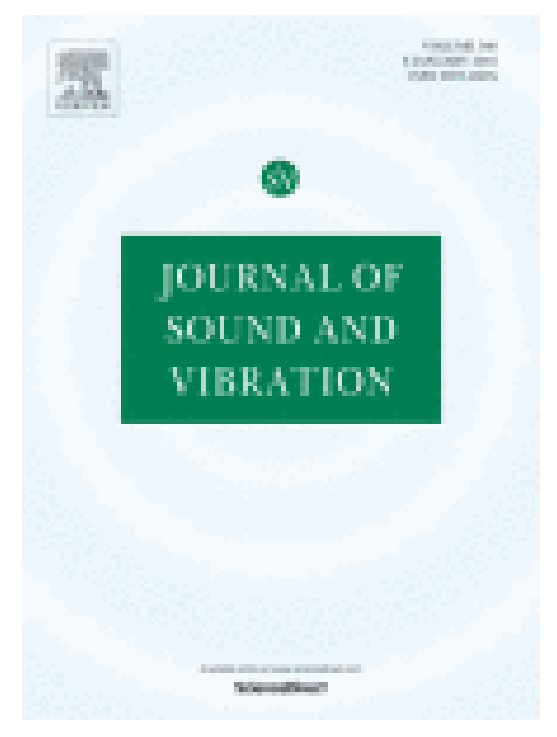

Title: "Unbalanced machinery vibration isolation with a semi-active pneumatic suspension"

Authors: Nieto A.J., Morales A.L., Chicharro, J.M., Pintado P.

JOURNAL OF SOUND AND VIBRATION, 329, pp. 3-12 (2010)

Keywords:

Pneumatic suspension, stiffness, transmissibility, unbalanced

DOI: 10.1016/j.jsv.2009.09.001 


\title{
Unbalanced machinery vibration isolation with a semi-active pneumatic suspension
}

\author{
A.J. Nieto ${ }^{1}$, A.L. Morales, J.M. Chicharro, P. Pintado \\ Área de Ingeniería Mecánica, E.T.S.I. Industriales (Universidad de Castilla - La \\ Mancha), Avda. Camilo José Cela s/n, 13071, Ciudad Real (Spain)
}

\begin{abstract}
The problem of unbalanced machinery isolation is tackled in this paper. The proposed solution incorporates an air suspension that can be adapted depending on the turning frequency. The system is built with three main parts: an air spring, a reservoir and a connecting pipe. A model of the suspension excited by the unbalanced rotor is also shown in this paper. The properties of the system make it possible to use a configuration of the suspension (one pipe size) over a bandwidth range and another configuration (another pipe size) over the remaining bandwidth range. This idea is implemented with solenoid controlled valves and the results show significant improvements with respect to completely passive configurations.
\end{abstract}

Key words: unbalanced rotor, attenuation, vibration, start-stop process PACS: 47.15.K-, 91.55.Ax, 47.27.nf

Email address: AntonioJavier.Nieto@uclm.es (A.J. Nieto) 


\section{NOMENCLATURE}

$\begin{array}{ll}A_{s} & \text { Spring effective area }\left[\mathrm{m}^{2}\right] \\ C_{r} & \text { The pipe restriction coefficient }\left[\mathrm{N}^{5} / \mathrm{m} / \mathrm{s}\right] \\ D_{p} & \text { The pipe's cross section diameter }[\mathrm{m}] \\ e_{0} & \text { Eccentricity of the unbalancing mass }[\mathrm{m}] \\ F & \text { Force exerted at the air spring }[\mathrm{N}] \\ g & \text { Acceleration due to gravity }\left[\mathrm{m} / \mathrm{s}^{2}\right] \\ K & \text { Pneumatic suspension total linear stiffness }[\mathrm{N} / \mathrm{m}] \\ K_{A S} & \text { Air spring effective area linear stiffness }[\mathrm{N} / \mathrm{m}] \\ K_{S} & \text { Air spring linear stiffness }[\mathrm{N} / \mathrm{m}] \\ K_{V S} & \text { Air spring volume linear stiffness }[\mathrm{N} / \mathrm{m}] \\ K_{V S R} & \text { Pneumatic suspension volume linear stiffness }[\mathrm{N} / \mathrm{m}] \\ l_{p} & \text { The pipe length }[\mathrm{m}] \\ m_{0} & \text { Unbalancing mass }[\mathrm{kg}] \\ M & \text { Sprung mass }[\mathrm{kg}] \\ P_{r} & \text { Relative pressure at the reservoir }[\mathrm{bar}] \\ P_{s} & \text { Relative pressure at the air spring }[\mathrm{bar}] \\ V_{r} & \text { Reservoir volume }\left[\mathrm{m}^{3}\right] \\ V_{s} & \text { Air spring volume }\left[\mathrm{m}^{3}\right] \\ V_{s r} & \text { Reservoir plus spring volume }\left[\mathrm{m}^{3}\right] \\ x & \text { Absolute response }[\mathrm{m}] \\ z & \text { Suspension height }[\mathrm{m}] \\ z & \text { Initial height for the air spring }[\mathrm{m}] \\ \gamma & \text { Specific heat ratio } \\ \mu & \text { Dynamic viscosity of air }[\text { Pa.s }] \\ \omega_{r o t} & \text { Rotor frequency }[\mathrm{rad} / \mathrm{s}] \\ \omega & \text { Low stiffness suspension frequency }[\mathrm{rad} / \mathrm{s}] \\ \omega_{s} & \text { High stiffness suspension frequency }[\mathrm{rad} / \mathrm{s}] \\ \omega_{t r} & \text { Transition frequency }[\mathrm{rad} / \mathrm{s}] \\ & \end{array}$

\section{Introduction}

Rotating motors and components are commonplace in industrial machinery. Unbalanced rotating masses are usually the origin of undesirable vibrations. Sometimes, the identification and correction of such a problem is easy by means of re-balancing. Nevertheless, there are cases in which this operation is not so simple. For instance, the unbalanced machine part may 
be difficult to reach or there may be no certainty about the origin of the vibration.

The problem has been studied from many different angles. Some authors [1] researched about elastodynamic models of coupled bending and torsional systems using the Lagrangian approach. Other works in this same direction study a model for coupled torsional and lateral vibrations of unbalanced rotor with rotor-to-stator rubbing using Lagrangian dynamics $[2,3]$. Other analytical methods analyze the instability and mechanical unbalanced response of induction motors considering unbalanced electromagnetic force produced in the induction motors with rotor eccentricity [4]. Recent developments in active control make it possible to control the dynamic behaviour of mechanical systems controlling discrete structures [5] or flexible structures [6]. Others tried correcting unbalances during operation in steady state and transient responses. The method consists of generating a correction force using two mobile weights situated in the same plane and running at a constant radius of the rotation axis [7].

The dynamics and stability of a rotating system have been improved with electromagnetic non-contact dampers in works like [8] and [9]. Other improvements in the dynamic performance of rotor-shaft systems with resonance problems are achieved in [10]. Polymeric material shaped as sectors has been considered as bearings. Polymeric materials have been considered for stiffness variation with the frequency of excitation. Optimization of thickness and length has also been pursued. Magnetic bearings are used to augment system damping and actively control the first critical speed in [11]. However, this type of control is not able to control higher order vibration modes.

In this paper, a pneumatic suspension is used with the aim of isolating vibrations from unbalanced rotors. An analytical model is built for this pneumatic system in order to simulate the behaviour of the system. Some tests were carried out to validate the simulations and a control procedure is proposed to avoid resonant areas when the machinery is working at variable speed. Good agreement is found between simulations and experiment and the proposed strategy is capable of significantly improving performance.

\section{Rotor-suspension analytical model}

\subsection{Rotor-suspension system}

The scheme of the rotor-suspension system can be seen in Fig. 1. This system is based on a configuration previously studied by the authors [12]. 
The pneumatic suspension consists of three principal parts: an air spring, a rigid tank and a set of two pipes connecting the first two elements via solenoid valves (one per pipe). A wheel with a little mass located in its periphery is connected to the rotor shaft. The rotor turns with rising frequency $\omega_{\text {rot }}$ and, as a result, the sprung mass oscillates vertically. The model tries to predict sprung mass response as a function of rotor frequency $\omega_{\text {rot }}$.

Three assumptions have been made for the closed system that includes the air spring and reservoir. The first of them is the assumption of isothermal compression and expansion of the air. The second is the assumption that the air behaves as an ideal gas. The last assumption is the consideration of an incompressible-fully-developed-laminar flow (Hagen-Poiseuille flow), in particular, Mach number $M<0.3$ and Reynolds number $R e<2300$, which is reasonable taking into account the size of the pipes and the amplitude and frequency of the excitation signal [13].

The governing equations of the system are shown in Eq. (2). The first of them is the continuity equation that assesses the mass flow from the air spring to the reservoir. The second one has into account the discharge process that happens from the reservoir to the air spring. The size of the pipe is involved in this process and a restriction coefficient $\left(C_{r}\right)$ is defined to quantify that influence [14]:

$$
C_{r}=\frac{\pi D_{p}^{4}}{128 \mu l_{p}}
$$

where $l_{p}$ is the pipe length, $D_{p}$ is the pipe cross section diameter, and $\mu$ the dynamic viscosity of air. The third equation evaluates the force exerted by the air spring as a function of the air spring effective area and pressure. Finally, the system dynamics is governed by Newton's second law (taking into account the unbalanced force). The system of differential equations is:

$$
\left\{\begin{array}{l}
\dot{P}_{s}=-\dot{P}_{r} \frac{V_{r}}{V_{s}}-V_{s}^{\prime} \frac{P_{s}}{V_{s}} \dot{x} \\
\dot{P}_{r}=-\frac{\gamma C_{r}}{2 V_{r}}\left(P_{r}^{2}-P_{s}^{2}\right) \\
\dot{F}=\dot{P}_{s} A_{s}+P_{s} A_{s}^{\prime} \dot{x} \\
M \ddot{x}+M g-F=m_{0} e_{0} \omega_{r o t}^{2} e^{i w t}
\end{array}\right.
$$

where the unknown functions of time are the pressure at the air spring $\left(P_{s}\right)$, the pressure at the reservoir $\left(P_{r}\right)$, the exerted force $(F)$ and the absolute displacement $(x)$. The rest are known parameters like the sprung mass $(M)$, the reservoir volume $\left(V_{r}\right)$ and the restriction coefficient $\left(C_{r}\right)$; or functions that have to be determined experimentally like the air spring effective area 
$\left(A_{s}(z)\right)$ and volume $\left(V_{s}(z)\right)$ as functions of the air spring height $z$. The apostrophe above $A_{s}(z)$ and $V_{s}(z)$ denotes the derivative of these functions with respect to the air spring height $z$.

A first-order Taylor series expansion, similar to the one used in the pneumatic suspension in [12], is applied to the above differential equations to obtain a linear version. The resulting equations are written as:

$$
\left\{\begin{array}{l}
\dot{P}_{s}=-\dot{P}_{r} \frac{V_{r}}{V_{s}^{s t}}-\kappa \frac{P_{s}^{s t}}{V_{s}^{s t}} \dot{x} \\
\dot{P}_{r}=-\frac{\gamma C_{r} P_{s}^{s t}}{V_{r}}\left(P_{r}-P_{s}\right) \\
\dot{F}=A_{s}^{s t} \dot{P}_{s}+\lambda P_{s}^{s t} \dot{x} \\
-M \omega_{r o t}^{2} x-F=m_{0} e_{0} \omega_{r o t}^{2}
\end{array}\right.
$$

where the superscript st denotes the static equilibrium point (around which the linear version of the model is built), and the parameters $\lambda$ and $\kappa$ are, respectively, $\lambda=A_{s}^{\prime s t}$ and $\kappa=V_{s}^{\prime} s t$.

The last equation in system Eq.(3) is obtained from the time derivative of the last equation in system Eq.(2) assuming a harmonic solution for both $x(t)$ and $F(t)\left(x(t)=X e^{i w t}\right.$ and $\left.F(t)=F e^{i w t}\right)$ in order to simplify that equation. The Laplace transform of the linear system yields:

$$
\left\{\begin{array}{l}
s P_{s}(s)+\frac{V_{r}}{V_{s}^{s t}} s P_{r}(s)+\kappa \frac{P_{s}^{s t}}{V_{s}^{s t}} s(s)=0 \\
s P_{r}(s)+\frac{\gamma C_{r} P_{s}^{s t}}{V_{r}}\left(P_{r}(s)-P_{s}(s)\right)=0 \\
s F(s)-A_{s}^{s t} s P(s)-\lambda P_{s}^{s t} s X(s)=0 \\
-M \omega_{r o t}^{2} X(s)-F(s)-m_{0} e_{0} \omega_{\text {rot }}^{2}=0
\end{array}\right.
$$

Rearranging the above equations one may obtain the transfer function between the exerted force $\mathrm{F}(\mathrm{s})$ and the sprung mass response $\mathrm{X}(\mathrm{s})$, that is:

$$
H(s)=\frac{F(s)}{X(s)}=-\frac{s\left(K_{A S}+K_{V S}\right)+W K_{V S}\left(1+\frac{K_{A S}}{K_{V S R}}\right)}{s+W\left(\frac{K_{V S}}{K_{V S R}}\right)}
$$

where $W$ is defined as $W=\gamma C_{r} P_{s}^{s t} / V_{r}$, and the linear stiffness terms $K_{A S}$, $K_{V S}$, and $K_{V S R}$ are defined as:

$$
\begin{gathered}
K_{A S}=-P_{s}^{s t} \lambda \\
K_{V S}=\frac{P_{s}^{s t} A_{s}^{s t}}{V_{s}^{s t}} \kappa
\end{gathered}
$$




$$
K_{V S R}=\frac{P_{s}^{s t} A_{s}^{s t}}{V_{s}^{s t}+V_{r}} \kappa
$$

The sprung mass response written in the Laplace domain, using the linearized equations, can be written as follows:

$$
X(s)=-m_{0} e_{0} \omega_{r o t}^{2}\left(\frac{s+W \frac{K_{V S}}{K_{V S R}}}{s\left(K_{A}+K_{V S}-\omega_{r o t}^{2} M\right)+\left(W K_{V S}\left(1+\frac{K_{A}}{K_{V S R}}\right)-\omega_{r o t}^{2} M W \frac{K_{V S}}{K_{V S R}}\right)}\right)
$$

or, in a normalized version as:

$$
X(s)\left[\frac{M}{m_{0} e_{0}}\right]=-\frac{s \omega_{r o t}^{2}+J \omega_{r o t}^{2}}{s\left(\omega_{s}^{2}-\omega_{r o t}^{2}\right)+J\left(\omega^{2}-\omega_{r o t}^{2}\right)}
$$

where $J=W\left(K_{V S} / K_{V S R}\right), \omega_{s}=\sqrt{K_{S} / M}, \omega=\sqrt{K / M}, K_{S}$ being $K_{A S}+$ $K_{V S}$ and $K$ being $K_{A S}+K_{V S R}$. The linear stiffnesses $K_{S}$ and $K$ correspond respectively to the upper and lower stiffness limits as was analyzed in detail in [12]. The upper limit in related to relatively high excitation frequencies and the lower one with relatively low frequencies.

Other important magnitude to study is the force transmitted to the foundation, which can be obtained from the previous equations. Combining the fourth equation in system (4) and the transfer function (5), the transmitted force yields:

$$
F(s)=\left[\frac{m_{0} e_{0} \omega_{r o t}^{2}+F(s)}{M \omega_{r o t}^{2}}\right] H(s)
$$

$H(s)$ being the transfer function between the transmitted force and the sprung mass displacement given in Eq. (5). The following normalized transfer function is obtained after rearranging terms:

$$
\frac{F_{t r}}{F_{c e n t}}=\frac{F(s)}{m_{0} e_{0} \omega_{\text {rot }}^{2}}=\left[\frac{H(s)}{M \omega_{r o t}^{2}-H(s)}\right]
$$

which can also be written as:

$$
\frac{F_{t r}}{F_{c e n t}}=\frac{s \omega_{s}^{2}+J \omega_{r o t}^{2}}{s\left(\omega_{r o t}^{2}-\omega_{s}^{2}\right)+J\left(\omega_{r o t}^{2}-\omega^{2}\right)}
$$

This is the transfer function of the transmitted force normalized with the centrifugal force generated by the unbalanced rotor. 


\subsection{Analytical results}

The analytical model in the previous section is used next in order to simulate the system response. To this end, the air spring effective area and volume $\left(A_{s}(z)\right.$ and $\left.V_{s}(z)\right)$ need to be experimentally characterized. This is done by placing the air spring in a hydraulic load unit and filling it with air up to an initial pressure that is kept constant throughout the test. The air spring height is modified with the hydraulic actuator and functions $A_{s}(z)$ and $V_{s}(z)$ are determined. More details of this test can be found in [12]. The results are shown in Fig. 2. The test is repeated for three values of pressure (2, 3 and 4 bar). The effect of pressure variation in both the effective area and volume is negligible.

A summary of the values of parameters used in this simulation is gathered in Table 1. The air spring and the reservoir volume have been chosen as small and as large as possible, respectively, in order to increase the ratio $K_{S} / K$. Figure 3 shows the normalized frequency response of the sprung mass obtained in the simulation. Two values of coefficient $C_{r}$ are used, $10^{-8}$ $N^{5} s / m$ for the smaller value (the so-called configuration 1) and $10^{-5} N^{5} \mathrm{~s} / \mathrm{m}$ for the larger one (the so-called configuration 2). As can be seen in this figure, configuration 2 (represented with a thick solid line) is less stiff than configuration 1 (represented with a thin solid line) and, therefore, its resonant frequency is lower. Nevertheless, the normalized peak response of the system is higher when using configuration 2, i.e., the damping ratio is also lower. The normalized force transmitted to the foundation by the rotor-suspension system is plotted in Fig. 4. The figure shows that there is no amplification of the dynamic force at very low frequencies, whereas no dynamic force is transmitted for very high frequencies. Peak values correspond to resonant frequencies that coincide with those shown in Fig. 3.

Fig. 5 shows the time response for configuration 1 and 2 when the rotor speeds up from 0 to $10 \mathrm{~Hz}$ at a rate of $0.05 \mathrm{~Hz} / \mathrm{s}$. The system physical behaviour can be easily understood by analysing Eq. (9) or its normalized version shown in Eq. (10). At low frequencies $\omega_{\text {rot }}$ is close to zero, and the numerators in both expressions are therefore almost zero. The mass displacement starts from a value of zero at very small rotor frequencies and raises as the rotor frequency grows. At high frequencies, $\omega_{\text {rot }}$ is quite large compared with $\omega$ and $\omega_{s}$, and, therefore, the response is very close to the constant limiting value. At intermediate frequencies, the value of $\omega_{\text {rot }}$ may coincide with either $\omega_{s}$ or $\omega$, cancelling either the first or the second term of the denominator of Eq. (10). These two frequencies correspond to the 
suspension resonant frequencies of both configurations 1 and 2. Configuration 2 experiences a less damped earlier resonance than configuration 1.

\subsection{Pneumatic suspension control strategy}

The previous analytical results suggest an adaptative suspension control strategy. The two curves in Fig. 3 correspond to two values of coefficient $C_{r}$ while the rest of parameters remain constant. An improved response can be achieved by switching between the two configurations, that is, taking configuration 1 up until frequency $\omega_{t r}$ (Fig. 3) and configuration 2 for higher frequencies. The frequency where the two curves cross (called transition frequency $\omega_{t r}$ ) can readily be obtained as:

$$
w_{t r}=\frac{1}{2} \sqrt{2 \omega_{s}^{2}+2 \omega^{2}}
$$

Any other curve drawn in that diagram, using any other $C_{r}$ coefficient, crosses all other at the same transition frequency $\omega_{t r}$. The modulus of the dynamic amplification at this frequency is:

$$
\left|\frac{X M}{m_{0} e_{0}}\right|_{t r}=\frac{K_{S}+K}{K_{S}-K}
$$

To implement the switching control, one would then need at least two pipes: one with the smallest value of the coefficient $C_{r}$ and one with the largest. The transition point indicates where a change from using one pipe to using the other must be made. A switching valve would have to be used for this task, selecting the smaller $C_{r}$ coefficient pipe if the excitation frequency is less than $\omega_{t r}$, and the larger one otherwise.

In this adaptative suspension the peak amplification occurs at frequency $\omega_{t r}$. Therefore, to optimize the suspension, one may try to achieve the smallest possible value of the transmissibility modulus corresponding to frequency $\omega_{t r}$. The modulus, as Eq. (15) shows, is a function of linear stiffness values $K_{S}$ and $K$. The selection of the suspension elements must be aimed at increasing $K_{S}$ and reducing $K$ or, in other words, one must seek the greatest distance possible between the eigenfrequency values corresponding to the two $C_{r}$ coefficients available. This involves reducing the air spring volume $V_{s}$ and increasing the reservoir volume $V_{r}$. 


\section{Experimental validation}

\subsection{Experimental workbench}

The experimental rig built for the rotor-suspension model validation can be seen in Fig. 6. A two-bellow air spring, with $80 \mathrm{~mm}$ total stroke, made of reinforced rubber (SBR), and functional from $-40^{\circ} \mathrm{C}$ to $70^{\circ} \mathrm{C}$ and up to 8 bar (model PM/31048 of Norgren Pneumatics) is used. The minimum and maximum strokes are 65 and $145 \mathrm{~mm}$, respectively. The second component is a $24 \mathrm{l}$ capacity steel tank. And the third is a pair of nylon pipes connecting the first two elements. The first pipe is $2 \mathrm{~m}$ long and has $2.7 \mathrm{~mm}$ of cross section diameter. The second one is $0.5 \mathrm{~m}$ long and its cross section diameter is $7.5 \mathrm{~mm}$. Two solenoid switching valves are used (one per pipe) to allow (or to block) the air flow between the air spring and the reservoir.

The sprung mass is located over the air spring. This mass comprises several parts: the first one is a steel open box enclosing a $2 \mathrm{CV}$ double shaft electric motor along with a $200 \mathrm{~mm}$ diameter steel wheel assembled at either end of the shaft (Fig. 6). A little mass of 30 grams is fixed on each wheel at a radial distance of $165 \mathrm{~mm}$ from the axis of rotation. Weights are placed on the steel box to complete a total sprung mass of $115 \mathrm{~kg}$. A linear ball bearing between the metal frame and the sprung mass is used to ensure its vertical displacement (Fig. 6).

The sprung mass displacement was measured with an LVDT (Schaevitz DC-EC 2000) with $\pm 50 \mathrm{~mm}$ measurement range and $0.01 \mathrm{~mm}$ resolution. The rotor speed is measured by an encoder (Baumer electric BRIH 40) with 1024 pulses per revolution. A data acquisition equipment is used to record data from the sensors. The same hardware was also used to send digital signals to the solenoid switching valves.

\subsection{Experimental results}

The first test deals with the sprung mass response when the motor undergoes start and stop processes. The electrical motor is programmed by computer to rev up from 0 to $10 \mathrm{~Hz}$ and back to $0 \mathrm{~Hz}$ at a rate of $0.05 \mathrm{~Hz} / \mathrm{s}$. The initial spring pressure is 2 bar, and the initial air spring height is $105 \mathrm{~mm}$. For this test, we switch the solenoid valves in order to keep one pipe open and the other one closed. When the rotor has swept all frequencies of the scheduled bandwidth, the solenoid valves are reversed, that is, the one that was opened must now be closed and viceversa. The results of this first test can be seen in Fig. 7, where the normalized displacement of the sprung mass 
is plotted as a function of the rotor angular frequency for the two pipes. The frequency response is represented with squares for the long and narrow pipe (configuration 1) and with circles for the short and wide pipe (configuration 2 ). These results are plotted along with the ones obtained with the simulation, with which they are seen to be in good agreement. The experimental resonant frequencies are located at 2.67 and $4.15 \mathrm{~Hz}$ for configurations 2 and 1 , respectively, whereas these same frequencies were found at 2.55 and 4.05 $\mathrm{Hz}$ when simulating the model.

Figure 8 shows the sprung mass time response when revving up the unbalanced rotor from 0 to $10 \mathrm{~Hz}$ at a rate of $0.05 \mathrm{~Hz} / \mathrm{s}$. Figure 8(a) shows the sprung mass absolute displacement when using configuration 2 , whereas Figure 8(b) shows the displacement when using configuration 1. Figure 8(c) shows the sprung mass response when both pipes are used, that is, one for frequencies lower than $\omega_{t r}$ and the other pipe for higher frequencies. It can be seen that this operation strategy avoids resonant frequencies and reduces the response amplification. This figure shows better performance compared with figures $8(\mathrm{a})$ and $8(\mathrm{~b})$ where no controls are implemented. The time value when the switching happens is obtained taking into account the transition frequency, the starting frequency $(0 \mathrm{~Hz})$ and the sweep rate $(0.05 \mathrm{~Hz} / \mathrm{s})$. The switching between pipes is done approximately after $75 \mathrm{~s}$.

Another interesting issue to consider is the time delay at transients between the suspension configurations when changing from one to the other. Two experiments are carried out. The first tries to evaluate the time delay switching from configuration 2 to configuration 1 . The rotor begins to turn at the resonant frequency of configuration 2 and, after a few cycles, the pipes are switched. The sprung mass response is shown in Fig. 9(a). The estimated transient time between the steady state of both configurations is 0.35 $\mathrm{s}$, approximately. The second test uses the opposite pipes, that is, it switches from configuration 1 to configuration 2. The results are shown in Fig. 9(b), the transient time now being $0.55 \mathrm{~s}$.

\section{Conclusions}

A model of a rotor-suspension system has been presented in this work. The results of simulations are in good agreement with those obtained experimentally.

A transient frequency has been obtained from the model that divides the frequency bandwidth into two different areas. Switching between these two 
areas has been implemented in the laboratory with the use of two different pipes. The transient frequency does not depend on the unbalanced mass nor does it depend on its radial position. The only parameter that must be known in order to implement the procedure is the total sprung mass. Great improvements have been achieved by using this switching strategy.

Transient speeds when changing from a suspension configuration to the other have also been studied in this paper. The results show that transients are fast enough and that this technique is suitable for unbalanced machinery.

\section{Acknowledgement}

The authors are grateful for the support received from Regional Project PCI08-0082 entitled "Análisis y diseño de elementos activos para el control de vibraciones" financed by the Consejería de Educación y Ciencia (Junta de Comunidades de Castilla-La Mancha). The authors also want to thank Prof. Agustín García-Berrocal for his help with the literature search.

\section{References}

[1] M.A. Mohiuddin, Y.A. Khulief, "Coupled bending torsional vibration of rotors using finite element", Journal of Sound and Vibration, 223(2), (1999), 297-316.

[2] B.O. Al-Bedoor, "Transient torsional and lateral vibrations of unbalanced rotors with rotor-to-stator rubbing", Journal of Sound and Vibration, 229(3), (2000), 627-645.

[3] Z. Yuan, F. Chu, Y. Lin, "External and internal coupling effects of rotor's bending and torsional vibrations under unbalances", Journal of Sound and Vibration, 299, (2007), 339-347.

[4] B.S. Yang, Y.H. Kim, B.G. Son, "Instability and imbalance response of large induction motor rotor by unbalanced magnetic pull", Journal of Vibration and Control, 10, (2004), 447-460.

[5] J. Der Hagopian, L. Gaudiller, B. Maillard, "Hierarchical control of hydraulic active suspensions of a fast all-terrain military vehicle", Journal of Sound and Vibration, 222(5), (1999), 723-752. 
[6] L. Gaudiller, J. Der Hagopian, "Active control of flexible structures using a minimum number of components", Journal of Sound and Vibration, 193(3), (1996), 713-741.

[7] C. Alauze, J. Der Hagopian, L. Gaudiller, "Active balancing of turbomachinery: application to large shaft lines", Journal of Vibration and Control, 7, (2001), 249-278.

[8] Y. Kligerman, O. Gottlieb, M.S. Darlow, "Nonlinear vibration of a rotating system with an electromagnetic damper and a cubic restoring force", Journal of Vibration and Control, 4, (1998), 131-144.

[9] B.O. Al-Bedoor, "Modeling the coupled torsional and lateral vibrations of unbalanced rotors", Computer methods in applied mechanics and engineering, 190, (2001), 5999-6008.

[10] J.K. Dutt, T. Toi, "Rotor vibration reduction with polymeric sectors", Journal of Sound and Vibration, 262, (2003), 769-793.

[11] Z. Abduljabbar, M.M. ElMadany, A.A. AlAbdulwahab. "Active vibration control of a flexible rotor", Computers and Structures, 58(3), (1996), 499-511.

[12] A.J. Nieto, A.L. Morales, A. González, J.M. Chicharro, P. Pintado, "An analytical model of pneumatic suspensions based on an experimental characterization", Journal of Sound and Vibration, 313 (1-2), (2008), 290-307.

[13] C. Erin, B. Wilson, J. Zapfe, "An improved model of a pneumatic vibration isolator: theory and experiment", Journal of Sound and Vibration, 218 (1), (1998), 81-101.

[14] I.H.Shames, "Mechanics of fluids", Second edition. McGraw-Hill, 1982. 


\section{Figure captions}

Figure 1. Scheme used to study the unbalanced rotor vibration isolation with a pneumatic suspension.

Figure 2. Experimental characterization of the air spring, functions (a) $A_{s}(z)$ and (b) $V_{s}(z)$.

Figure 3. Normalized modulus of the sprung mass response obtained from the simulations (thin solid line for configuration 1 and thick solid line for configuration 2).

Figure 4. Normalized force transmitted to the foundation obtained from the simulations.

Figure 5. Sprung mass absolute displacement amplitude as a function of time for a rising-falling rotating frequency at a rate of $0.05 \mathrm{~Hz} / \mathrm{s}$ obtained from the simulations (thin solid line for configuration 1 and thick solid line for configuration 2).

Figure 6. Experimental workbench.

Figure 7. Comparison of model simulations with experimental results. Normalized modulus of the sprung mass response. Squares: configuration 1 experimental response; circles: configuration 2 experimental response; solid lines: simulation results.

Figure 8. Experimental results for the sprung mass absolute displacement amplitude as a function of time for a revving up motor at a rate of $0.05 \mathrm{~Hz} / \mathrm{s}$ : (a) configuration 2, (b) configuration 1 and (c) switching mode.

Figure 9. Experimental study of transients between the suspension configurations: (a) switching from configuration 2 to configuration 1 and (b) switching from configuration 1 to configuration 2 .

\section{Tables}

Table 1. Summary of parameters used in the analytical simulation of the rotor-suspension model. 
Table 1: Summary of parameters used in the analytical simulation of the rotor-suspension model.

\begin{tabular}{|c|c|c|c|}
\hline Parameter & Value & Parameter & Value \\
\hline$m_{0}$ & $0.030 \mathrm{~kg}$ & $A_{s}^{s t}$ & $4.5 \cdot 10^{-3} \mathrm{~m}^{2}$ \\
$e_{0}$ & $0.165 \mathrm{~m}$ & $\gamma$ & 1.4 \\
$M$ & $115 \mathrm{~kg}$ & $P_{s}^{s t}$ & $2 \mathrm{bar}$ \\
$V_{r}$ & $24 l$ & $\lambda$ & $-9 \cdot 10^{-2} \mathrm{~m}$ \\
$V_{s}^{s t}$ & $0.56 l$ & $\kappa$ & $9 \cdot 10^{-3} \mathrm{~m}^{2}$ \\
\hline
\end{tabular}

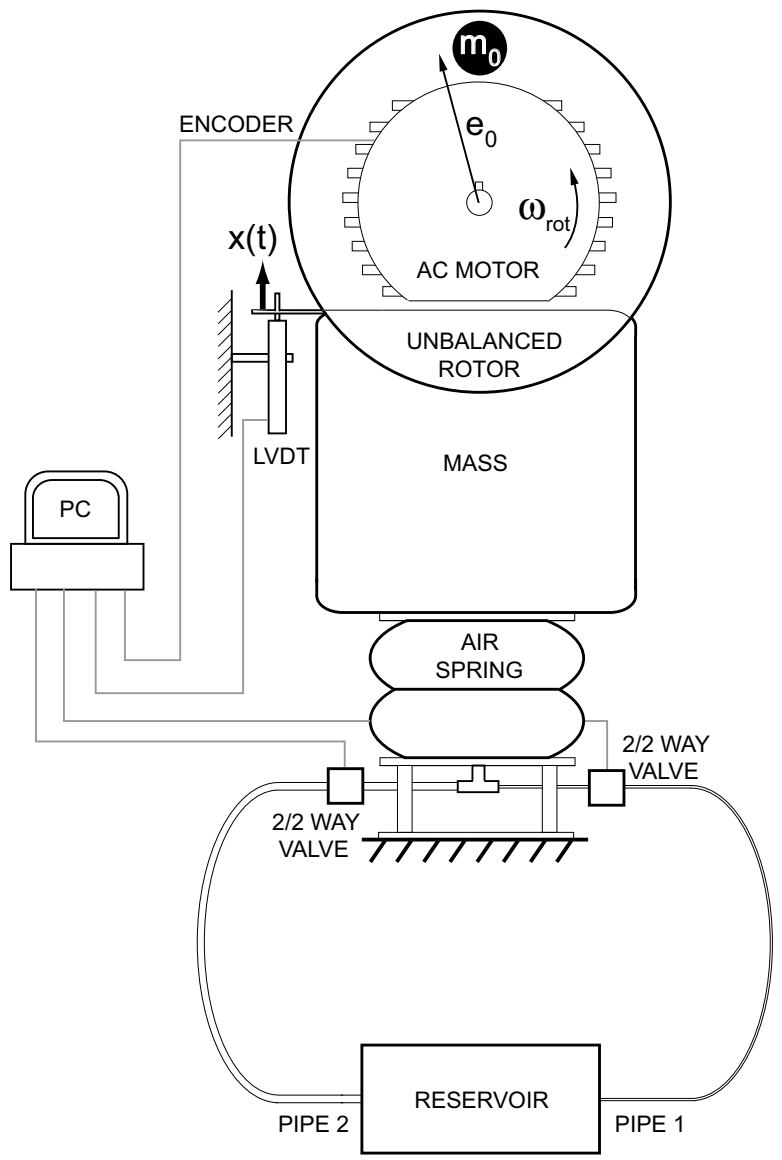

Figure 1: Scheme used to study the unbalanced rotor vibration isolation with a pneumatic suspension. 
(a)

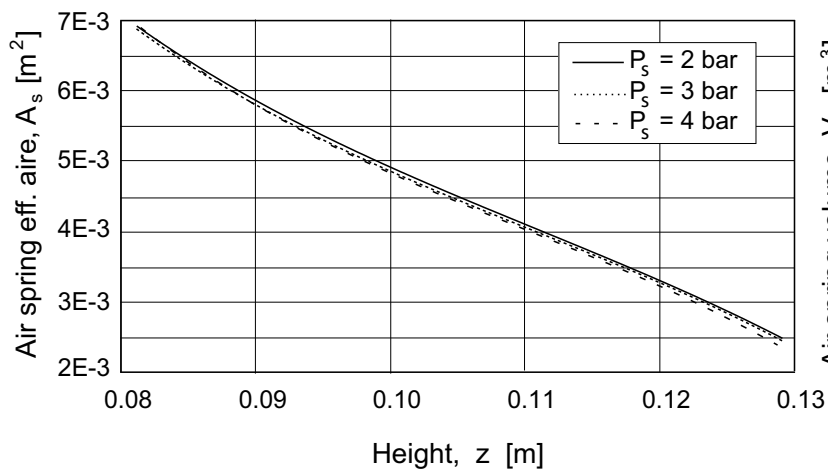

(b)

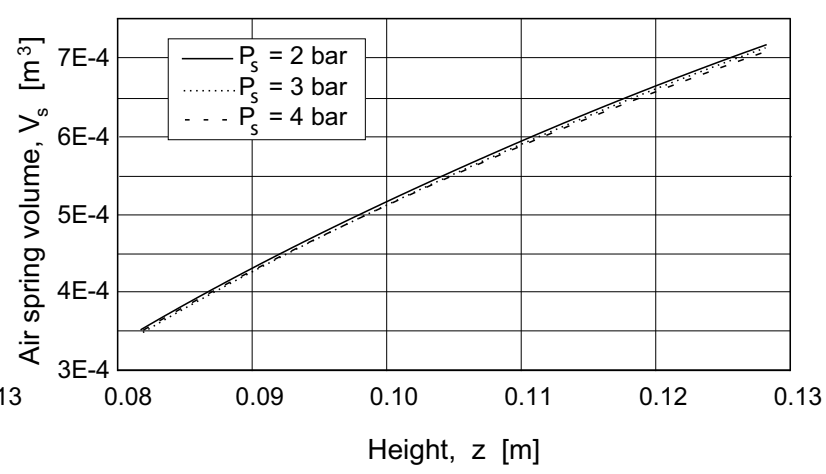

Figure 2: Experimental characterization of the air spring, functions (a) $A_{s}(z)$ and (b) $V_{s}(z)$

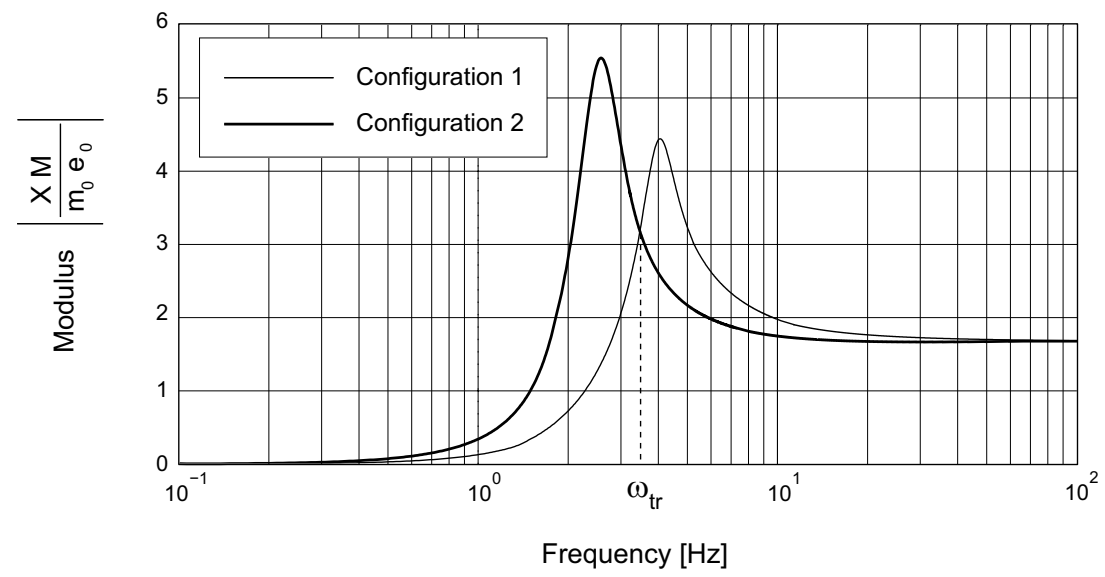

Figure 3: Normalized modulus of the sprung mass response obtained from the simulations (thin solid line for configuration 1 and thick solid line for configuration 2). 


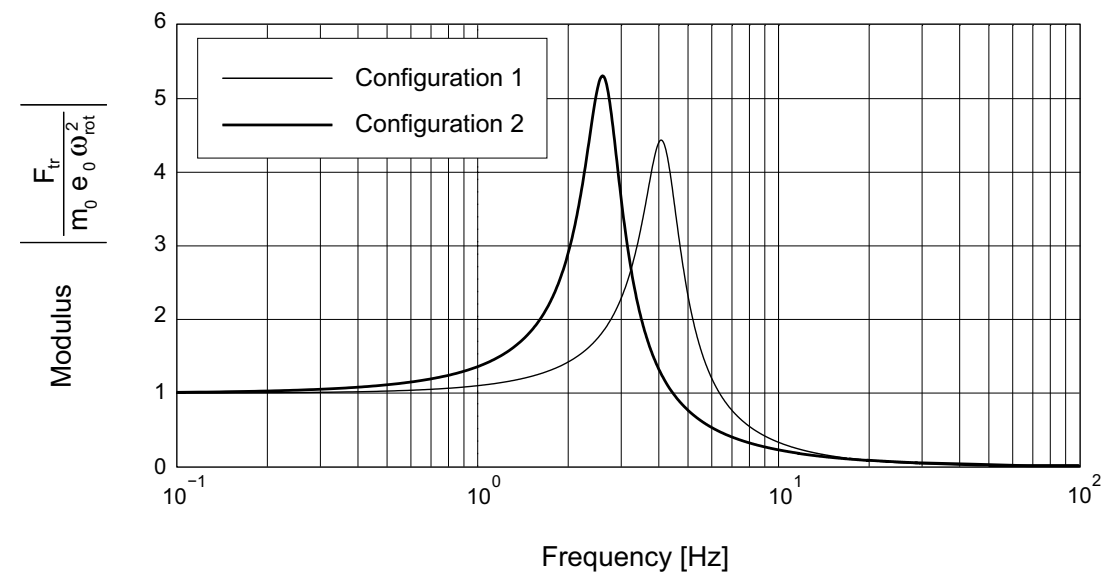

Figure 4: Normalized force transmitted to the foundation obtained from the simulations.

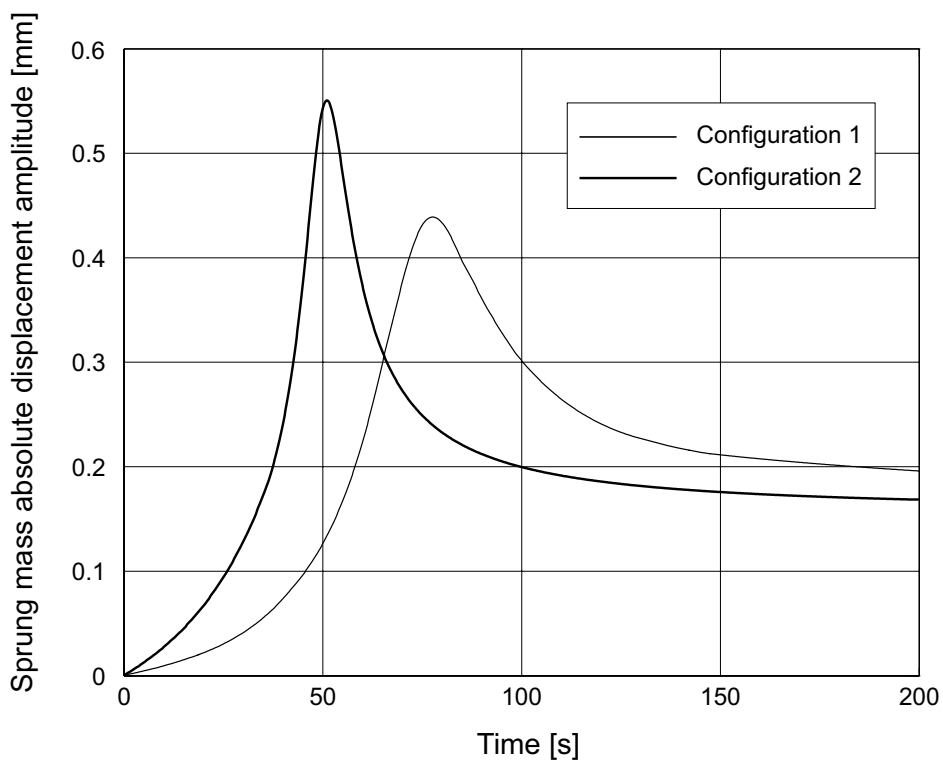

Figure 5: Sprung mass absolute displacement amplitude as a function of time for a risingfalling rotating frequency at a rate of $0.05 \mathrm{~Hz} / \mathrm{s}$ obtained from the simulations (thin solid line for configuration 1 and thick solid line for configuration 2). 


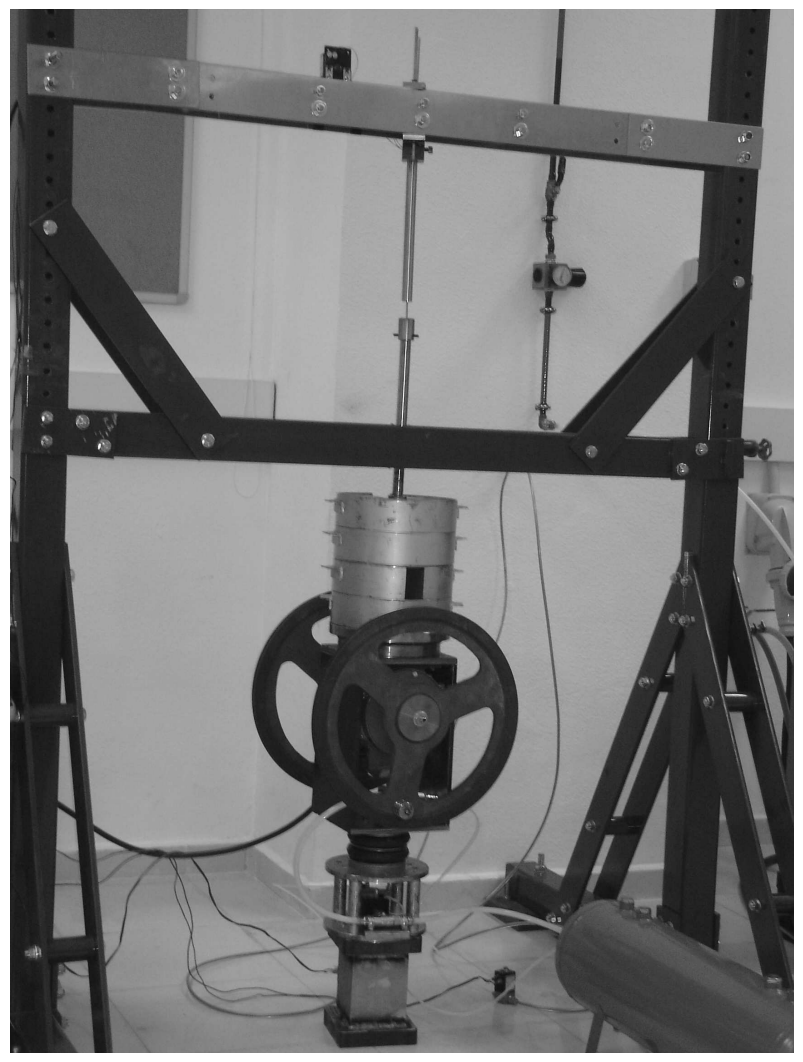

Figure 6: Experimental workbench. 


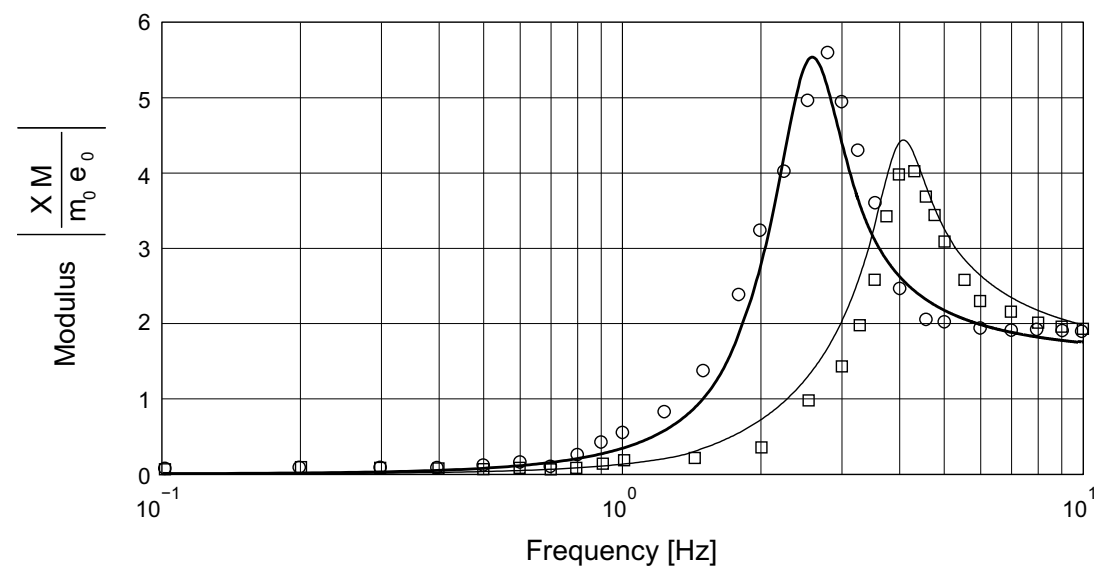

Figure 7: Comparison of model simulations with experimental results. Normalized modulus of the sprung mass response. Squares: configuration 1 experimental response; circles: configuration 2 experimental response; solid lines: simulation results. 
a)
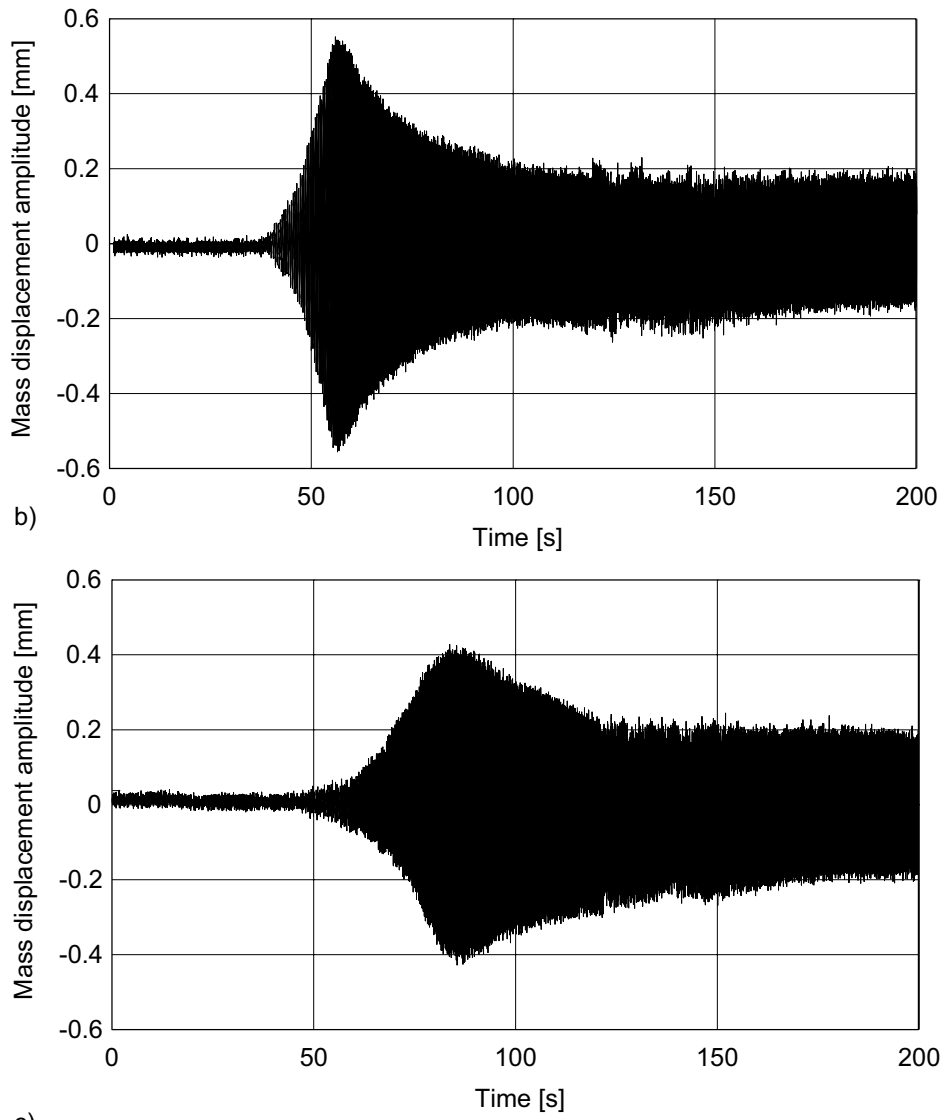

c)

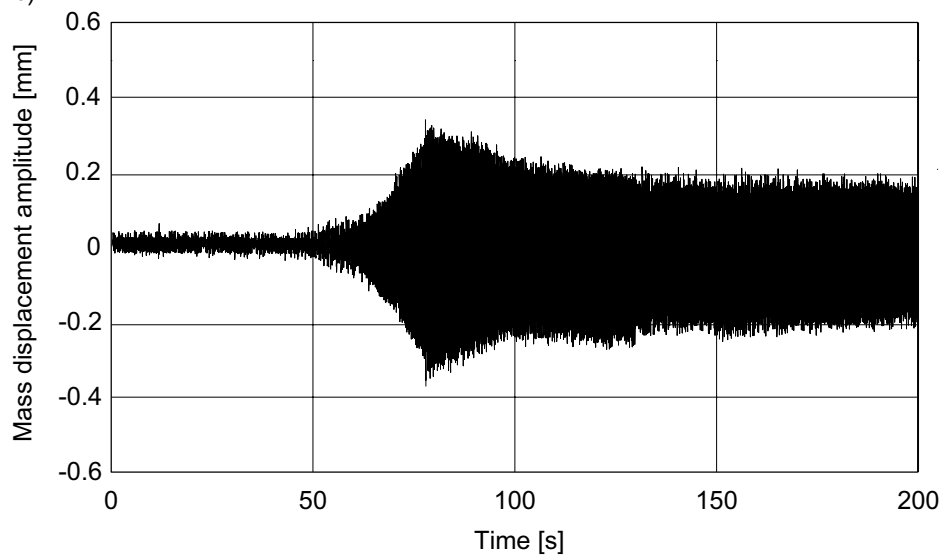

Figure 8: Experimental results for the sprung mass absolute displacement amplitude as a function of time for a revving up motor at a rate of $0.05 \mathrm{~Hz} / \mathrm{s}$ : (a) configuration 2, (b) configuration 1 and (c) switching mode. 


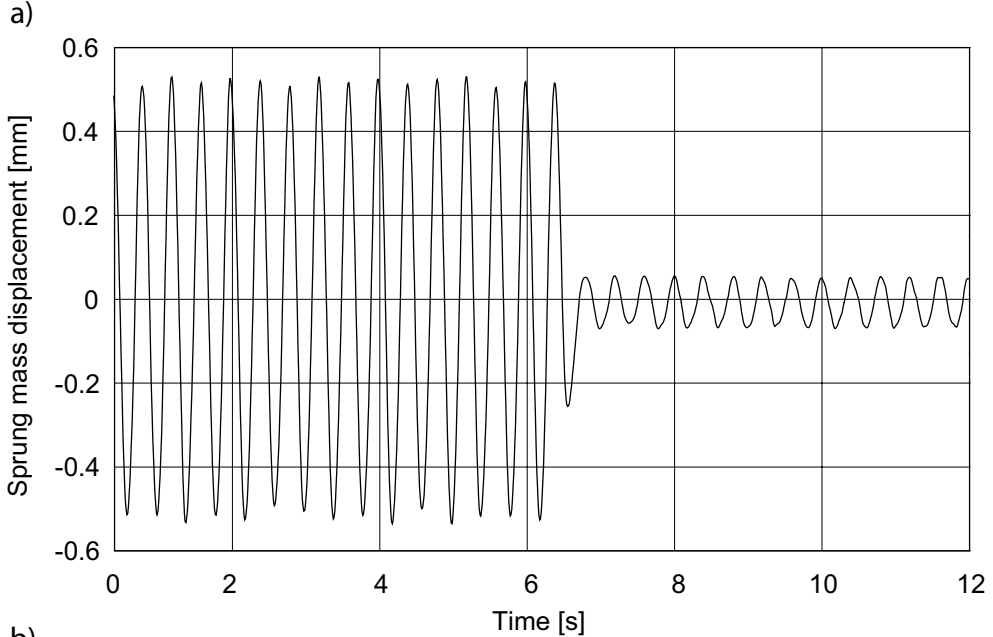

b)

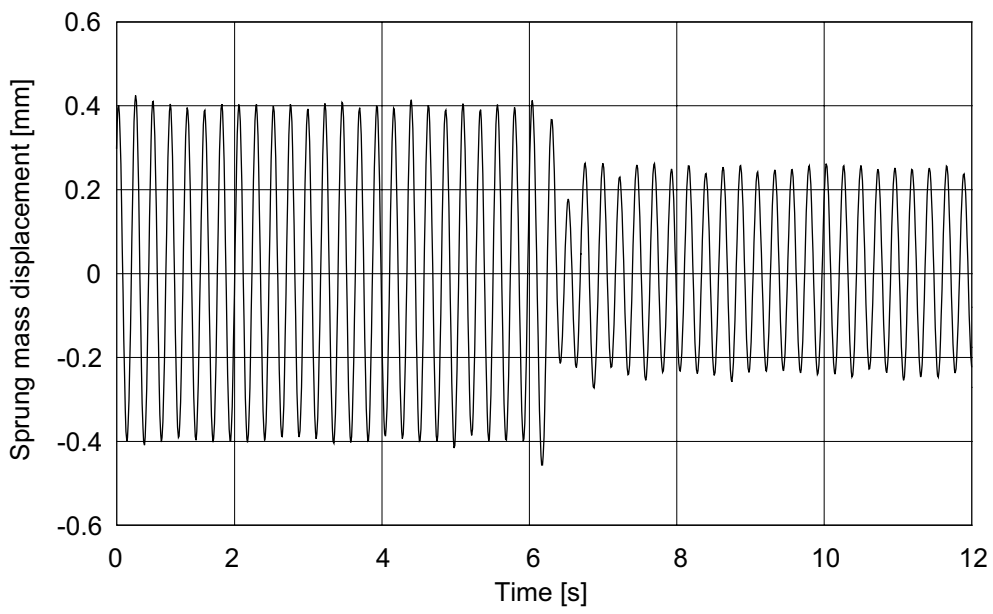

Figure 9: Experimental study of transients between the suspension configurations: (a) switching from configuration 2 to configuration 1 and (b) switching from configuration 1 to configuration 2 . 